\title{
Brake-control-based holistic truck motion control concept for automated driving
}

Dr. Falk Hecker, F. Stambrau, A. Mustapha, Knorr-Bremse Systeme für Nutzfahrzeuge GmbH

This manuscript is not available according to publishing restriction. Thank you for your understanding. 